\title{
2006-261: ACADEMIC - INDUSTRIAL PARTNERSHIPS TO ADVANCE POLLUTION PREVENTION
}

\section{Stewart Slater, Rowan University}

C. Stewart Slater is a Professor and Founding Chair of Chemical Engineering at Rowan University. He received his Ph.D., M.S. and B.S. from Rutgers University. His research and teaching interests are in the area of membrane technology where he has applied this to fields such as specialty chemical manufacture, green engineering, bio/pharmaceutical manufacture and food processing. He is the recipient of the 1999 Chester Carlson Award, 1999 and 1998 Joseph J. Martin Award, 1996 George Westinghouse Award, and the 1989 Dow Outstanding New Faculty Award.

\section{Mariano Savelski, Rowan University}

Mariano J. Savelski is Associate Professor of Chemical Engineering at Rowan University. He received his Ph.D. from the University of Oklahoma and B.S. from the University of Buenos Aires. His research is in the area of process design and optimization with over seven years of industrial experience. He has applied his expertise in water and energy integration in green engineering design to industrial projects from food processing to petroleum refining. He is also involved in research in sustainable fuels, examining ethanol production from biomass. He is the recipient of the 2000 Lindback Foundation Faculty Award.

\section{Robert Hesketh, Rowan University}

Robert P. Hesketh is a Professor and Chair of Chemical Engineering at Rowan University. He received his B.S. from the University of Illinois and Ph.D. from the University of Delaware. His research and teaching interests are in the area of reaction engineering, combustion kinetics and process engineering. He is the recipient of the 1999 Ray W. Fahien award, 2001 and 1998 Joseph J. Martin Award, 2002 Robert G. Quinn award, and the 1998 Dow Outstanding New Faculty Award. 


\title{
Academic - Industrial Partnerships to Advance Pollution Prevention
}

\begin{abstract}
Student projects have examined how to apply pollution prevention strategies to both R\&D and manufacturing in several chemical industries. This has been accomplished through industry-university partnerships with pharmaceutical and petrochemical companies. Several grants from the US Environmental Protection Agency have supported initiatives in green chemistry, engineering and design. These projects have the broader goal of supporting sustainability in the chemical industry.
\end{abstract}

\section{Introduction}

Too often the teaching of a technical subject like green engineering is limited to an individual class experience or one dimensional laboratory or design experience. The teaching of pollution prevention in the curriculum is greatly enhanced by active participation of students throughout the curriculum and in real-world projects. Green engineering is a multidisciplinary topic that if practiced to the fullest would greatly improve how industry operates and provide a sustainable future. Rowan University is incorporating green engineering into its curricula in various course and our latest efforts (as described in this paper) are to actively involve industry in green engineering projects through our engineering clinic program. In this paper we refer to the terms pollution prevention and green engineering interchangeably. Green chemistry and engineering methods are forms of pollution prevention. EPA defines pollution prevention/source reduction as any practice which does one or more of the following:

- Reduces the amount of any hazardous substance, pollutant, or contaminant entering any waste stream or otherwise released into the environment (including fugitive emissions) prior to recycling, treatment or disposal.

- Reduces the hazards to public health and the environment associated with the release of such substances, pollutants, or contaminants.

- Reduces or eliminates the creation of pollutants through

- increased efficiency in the use of raw materials, energy, water, or other resources; or

- protection of natural resources by conservation.

Specifically this paper addresses pollution prevention through green chemistry, green engineering and design for the environment strategies.

The EPA originally defined green engineering as the design, commercialization and use of processes and products that are feasible and economical while minimizing the generation of pollution at the source and also minimizing risk to human health and the environment [1]. The 
definition of green engineering was more broadly defined in a recent conference (Sandestin, Florida, 2003) to transforming existing engineering disciplines and practices to those that lead to sustainability. Green engineering incorporates development and implementation of products, processes, and systems that meet technical and cost objectives while protecting human health and welfare and elevating the protection of the biosphere as a criterion in engineering solutions [2]. Nine green engineering principles were developed as a result of this conference. Engineers should follow these principles to fully implement green engineering solutions:

- Engineer processes and products holistically, use systems analysis, and integrate environmental impact assessment tools.

- Conserve and improve natural ecosystems while protecting human health and well-being.

- Use life cycle thinking in all engineering activities.

- Ensure that all material and energy inputs and outputs are as inherently safe and benign as possible.

- Minimize depletion of natural resources.

- Strive to prevent waste.

- Develop and apply engineering solutions, while being cognizant of local geography, aspirations and cultures.

- Create engineering solutions beyond current or dominant technologies; improve, innovate and invent (technologies) to achieve sustainability.

- Actively engage communities and stakeholders in development of engineering solutions.

\section{Educational Approach}

The traditional and probably most common method of introducing aspects of green engineering has been through a senior and graduate level elective course on environmental engineering, with an emphasis on process treatment. Courses were developed that focus on methods to minimize or prevent waste streams from existing chemical plants in the 1990's. The educational progression mirrors the progression in industry. In industry initial efforts were applied to waste treatment whereas current efforts are aimed at reducing the total volume of effluent treated as well as the nature of the chemicals treated. Currently, many of the environmental and pollution prevention courses have been replaced by courses in green engineering, environmentally conscious chemical process engineering, and engineering for sustainable development [3]. These courses are typically offered during the senior year and are optional engineering courses. These stand-alone courses are excellent in providing detailed coverage of the subject and are needed in the engineering curriculum. Students may get the impression that green engineering is either optional or something done at the end of the design process, since this course is usually both optional and at the end of their undergraduate education.

A better method is to introduce these concepts throughout the curriculum [4] which helps to emphasize that engineers should be using green engineering and sustainability throughout the design process. Implementing this integrated approach, in which students see green engineering throughout their four years of engineering shows the importance of this subject to the students and reinforces the need to employ this subject in industry. Both of these methods of education should be encouraged and further development is needed. 
In 1998 a program was initiated with support from the EPA to develop a text book on green engineering, "Green Engineering: Environmentally Conscious Design of Chemical Processes" [5] by Allen and Shonnard; disseminate these materials and assist university faculty in using these materials through national and regional workshops coordinated with the American Society for Engineering Education (ASEE), Chemical Engineering Division [6]. Rowan University took the lead in developing curriculum modules that can be used to easily integrate green engineering concepts throughout the curriculum. These efforts include the development of instructor guides, case studies, homework problems and in-class examples. These efforts originally piloted at Rowan were expanded involving faculty from across the country in module development and implementation. These green engineering course modules can be found on the green engineering web site (www.rowan.edu/greenengineering) [7,8] and are described in several papers $[9,10,11]$.

\section{Pollution Prevention Projects}

We have sought to improve on our concept of green engineering education integration in a way that not only enhances student learning, but improves the environment. This is through real-world projects in our engineering clinic sequence [9]. The green engineering projects use a vertically integrated student team of junior, senior and masters level engineering students who work with the industrial partner on approaches to pollution prevention. These projects provide an illustration of how companies can take advantage of universities in their region to assist in sustainable development and manufacturing. Students are able to learn about green engineering design and apply their knowledge directly to a real industrial case. The industries involved partner by providing support in terms of senior staff, scientific/engineering process data, access to plant facilities, and take initiatives recommended forward to process improvements on the plant scale. The industries examined represent two distinct industrial sectors, thus providing student teams with unique challenges in designing for the environment. We expect these projects to yield the following green engineering outcomes which will be described in further detail in the paper.

\section{Petrochemical industry - Valero Energy Corp (Paulsboro, NJ)}

- energy and water reduction/optimization

- increase efficiency in use of raw materials and energy

- emissions reduction/wastewater minimization

\section{Pharmaceutical industry - Bristol-Myers Squibb Co (New Brunswick, NJ)}

- solvent reduction (in both quantities and toxicity)

- green engineering approach in initial phases of drug development to

$\circ$ increase efficiency in use of raw materials and energy

○ reduction in pollutant liquid waste

- energy conservation/recovery through life cycle assessment of processes

The role of academia in industrial projects in the green engineering area is vital to infuse fresh ideas into operations. The students and industrial personnel gain through training in the project briefings and plant visits where an exchange of ideas is encouraged. Each project starts 
with seminars by each party on their particular area of expertise thus fulfilling a goal of outreach. Students are encouraged to step back and look at the big picture and provide some points of view that draw upon both principles learned in prior courses or concepts learned in another clinic project. The clinic team will also develop a green engineering heuristic that will be applicable to other companies, thus expanding the outcomes of the projects.

Rowan University has experience in industrially-oriented projects through its Engineering Clinic program that partners academic experts with industry to solve real-world problems. Through the projects described in this paper we are extending our already successful program to pollution prevention and green manufacturing. Our record of success with industrial partners with clinic projects at Rowan has resulted in significant outcomes. $[12,13,14,15,16,17,18,19,20,21]$.

These projects are funded by grants from the U.S. Environmental Protection Agency, Region 2, through Pollution Prevention Grants Program and the Conservation Challenge Grants Program. Both of these competitive funding sources are administered by each region of the country. Matching and/or in-kind support is required for the pollution prevention grant program. Region 2, where Rowan University is located, is comprised of New Jersey, New York, Puerto Rico, the U.S. Virgin Islands and the Tribal Nations in those states.

\section{Petroleum Refining Industry Initiative}

Some of the largest and most visible manufacturing plants in EPA Region 2 are the petroleum refineries that are situated near the major transportation arteries in New Jersey. They are excellent candidates for applying green engineering methods because of their size, quantities of material processed and emissions issues. Since no new refineries have been built in the United States in over 25 years, the industry needs to take existing older facilities and upgrade and optimize them to allow operations to be conducted in an economic and environmental way. This upgrade is necessary since refining operations are vital to national security and energy policy.

The U.S. petroleum refining industry has come under considerable strain because of several important factors and changes in the industry. Over the years, there has been an increased domestic demand for petroleum products and a decrease in U.S. production; however, there has been no new capacity added. This lack of infrastructure growth has caused a tremendous strain on the industry in meeting existing demand, and the U.S. has had to increase the amounts of imports to meet these needs.

A number of Federal regulations such as the Clean Air Act and the Clean Water Act along with stringent state regulations have also caused the industry to incur high costs for environmental compliance. These costs are accrued because refineries must produce reformulated, cleaner-burning gasoline, which require companies to replace or modify existing equipment with devices for controlling emissions. These costs of compliance are having an effect on refineries trying to expand and to keep pace with the country's increasing demand. 
Through this project we are assisting industry in innovative and cost-effective pollution prevention strategies and improve their methods through on-site training and implementation. The initial exploratory phase of the project involves a series of brainstorming sessions with engineers from various refinery operations to look at challenges and opportunities in pollution prevention and green engineering.

A team of Rowan faculty and students is currently working side-by-side with Valero staff investigating various aspects of pollution prevention strategies. Some of the areas that we anticipate being involved in are wastewater minimization through water reuse and recycle and energy optimization though heat integration and heat exchanger network retrofitting $[12,13,14,15,16]$. This project required Rowan University to enter into a confidentiality agreement with Valero and therefore specific details regarding the project cannot be described. The overall energy optimization is a multi-step process that involves

- Simulation of existing units:

- Flowsheet simplication

- Determination of the basic energy savings horizons

- Synthesis of the horizon networks

- Retrofit network

- Test the feasibility of the new retrofit network

- Cost Analysis

The overall water use optimization will also be conducted using a methodology developed by Savelski [21]. In this case, the proposed procedure identifies the minimum water usage (target) of a given set of water-using/water-disposing processes (desalters, hydrodesulphurization processes, distillation, etc), and then determines a network of interconnections of water streams among the processes so that the overall fresh water consumption is minimized while the processes receive water of adequate quality. As with the energy optimization method, the water-use minimization involves several steps:

- Fresh Water Targeting

- Network Construction

- Alternative Networks

The results of the Spring semester project will be presented at the conference.

\section{$\underline{\text { Pharmaceutical Industry Initiatives }}$}

The pharmaceutical industry is a prominent commercial sector in EPA Region 2. The majority of the leading pharmaceutical companies have headquarters, research \& development centers and manufacturing facilities in New Jersey, New York and/or Puerto Rico. This industry is vital to the regional economy as well as to national security and the standard of living, health, and welfare of the public at large. For the United States pharmaceutical industry to grow and develop in a sustainable way (while at the same time meeting current and future EPA regulations), adoption of green engineering principles is necessary from R\&D through manufacturing. Through this project, a student-faculty team is working with Bristol-Myers 
Squibb scientists and engineers to develop green engineering protocols that can be adopted by the pharmaceutical industry.

The majority of drug products made through organic synthesis routes require many sequential reaction steps, large quantities of organic solvents (with varying degrees of toxicity), and are made in batch processes. All of the above are not optimal from a green engineering manufacturing standpoint. A significant reduction in the use of solvents, in terms of quantities and toxicity, can be made if investigations into the early stages (Phase I or II) of drug development are performed. It is important to work with drugs in Phase I or II of development, since these changes can be incorporated into the final manufacturing steps that are approved by the FDA. By Phase III, "synthesis lock" and "process lock" prevent innovations from being easily implemented. The team is working on developing a heuristic that pharmaceutical companies can follow in the development of new drugs.

The specific objectives of this project are to identify reductions in the use of hazardous chemicals in a drug synthesis. The Rowan team first met with the Bristol-Myers Squibb staff and discussed several possible drugs at various stages of development that we could examine. A confidentiality agreement was signed which limits the amount of information we can reveal about the drug and the manufacturing process. A cancer drug in the early stages of development was selected. The team then met to set and review project goals/objectives.

The initial part of the project involved a review of process development documentation and a pilot plant visit to understand equipment issues. The basic data on raw materials, products, byproducts of the process were analyzed. Green engineering metrics for lab-scale (discovery), intermediate and pilot-scale processes were compared. Life cycle assessment was made using overall material and energy balances along with environmental performance tools. Tier 1 tools such as economic criteria, environmental criteria, exposure limits, toxicity weighting in analyzing various drug production pathways.

Since organic solvents typically account for $80 \%$ of all chemicals in a pharmaceutical process, a significant part of the work focuses on process modifications to reduce solvents used. Several process opportunities for greener processes were explored. A life cycle assessment is conducted to compare these alternatives and show broader impacts on the ecosystem (greenhouse gas production, etc). These alternative production routes include new solvents and processing methods. Throughout this process, we are in constant contact with Bristol-Myers Squibb R\&D staff and get feedback for continuous improvement of our approach. The analyses of these alternative approaches are presented to the company in bimonthly progress meetings.

The process improvements made by the Bristol-Myers Squibb scientists have been quantified by the student and faculty team using several approaches. The student team investigated and applied metrics for process, safety and environmental impacts. This was done by comparing the resultant change in quantities of material and energy used at each process improvement. There is a broad category called processing metrics. The metrics in this category include mass, energy, water, and solvent usage along with waste management issues and solvent recovery. These factors are considered more frequently than the other metrics since these are the 
basic operating parameters of a chemical plant. All of these factors are typically used in pharmaceutical applications.

Students examined the mass metric which can be represented in numerous ways. Jimenez-Gonzalez et. al. used mass intensity as metric [22]. Mass intensity is the amount of total mass needed to make 1 kilogram or pound of a final API (active pharmaceutical ingredient) product. This metric can be found by using the yield for the upstream process, and the summation of the mass of solvents and water used in the process. Similarly solvent usage was another major parameter considered. Solvent intensity is defined as the amount of solvents including volatile organic compounds (VOCs) needed to make 1 kilogram of final pharmaceutical product. Waste intensity was yet another of the process metrics utilized.

Solvent selection and use was also measured by a unique solvent selection spreadsheet that quantifies all of the various process streams into one value that can represent the greenness of the process. Therefore the difference in process improvements for the different drug synthesis routes can be compared using one unifying index that combines various environmental and safety metrics such as TLV (threshold limit value), ingestion toxicity, biodegradation, aquatic toxicity, carcinogenicity, ozone depletion, global warming potential, half-life, smog formation, acidification, soil adsorption and bioconcentration factor. This spreadsheet uses a weighted scale based on the importance of the particular metric. In our case safety metrics such as TLV, ingestion toxicity and carcinogenicity, were weighted more heavily.

This overall index is useful when various process improvements are examined. For instance when a solvent washing step is eliminated or an alternate separation is proposed, these alternative can now be compared not only through traditional design protocols, but on a "green" scale as well $[23,24]$.

\section{Summary}

Two EPA-sponsored projects in pollution prevention have been started that focus on solving real-world industrial problems. These projects tackle pollution prevention issues of two distinctly different industry with similar goals of water, energy, and pollution reduction through green chemistry and engineering strategies. The students involved in these projects will be

analyzing process operations and examining methods to quantify material and energy usage and proposing approaches for green engineering design.

\section{Acknowledgements}

Support for the projects described in this paper are from the U.S. Environmental Protection Agency, Region 2:

- Conservation Challenge Grants Program X1 97275805-0

- Pollution Prevention Grants Program NP 97275905-0 


\section{References}

1 U.S. Environmental Protection Agency, Green Engineering web site http://www.epa.gov/opptintr/greenengineering/index.html

2 Ritter, S. K., "A Green Agenda for Engineering: New set of principles provides guidance to improve designs for sustainability needs," July 21, 2003, 81 (29) Chemical \& Engineering News pp. 30-32.

3 Hesketh, R.P., M. H. Gregg, C.S. Slater, "Green Engineering Education”, Chapter in Sustainability Science and Engineering: Principles, Elsevier Science Publishers, New York, 2006.

4 Hesketh, R.P. C. S. Slater, M.J. Savelski, K. Hollar, S. Farrell, “A Program to Help in Designing Courses to Integrate Green Engineering Subjects," International Journal of Engineering Education, 20(1), 113-123, 2004

5 Allen, D.T., D.R. Shonnard, Green Engineering: Environmentally Conscious Design of Chemical Processes, Prentice Hall, Englewood Cliffs, NJ (2001).

6 Cutlip, M. H.S. Fogler, C.S. Slater, "The ASEE Chemical Engineering Summer School for New Faculty," Proceedings of the 2003 Annual Conference of the American Society for Engineering Education , 2003.

7 Green Engineering Education Modules, Rowan University, www.rowan.edu/greenengineering

8. Hesketh, R.P., K. Jahan, A.J. Marchese, C.S. Slater, J.L. Schmalzel, T.R. Chandrupatla, R.A. Dusseau, "Multidisciplinary Experimental Experiences in the Freshman Engineering Clinic at Rowan University," Proceedings of the 1997 Annual Conference of the American Society for Engineering Education (1997).

9 Slater, C. S., R.P. Hesketh, J.A. Newell, S. Farrell, Z.O. Gephardt, M.J. Savelski, K.D. Dahm, B.G. Lefebvre, "Chemical Engineering at Rowan University,", Chemical Engineering Education, 39, 82-87, 2005.

10 Slater, C.S., R.P. Hesketh, D. Fichana, J. Henry, A.M. Flynn, M. Abraham, "Expanding the Frontiers for Chemical Engineers in Green Engineering Education" International Journal of Engineering Education, (in press 2006).

11 Slater C.S. and R.P. Hesketh "Incorporating Green Engineering into a Material and Energy Balance Course," Chemical Engineering Education 38(1), pp. 48-53, 2004.

12 Koppol A., Bagajewicz M., Dericks B. and Savelski M. J., "On Zero Water Discharge Solutions in the Process Industry", J of Environmental Sustainability, vol. 2, no. 2, 2004.

13 Savelski M. J. and Bagajewicz M., "On the Necessary Conditions of Optimality of Water Utilization Systems in Process Plants with Multiple Contaminants", Chemical Engineering Science, 58, no. 23-24, 2003.

14 Koppol A., Bagajewicz M., Dericks B., and Savelski M. J., "On Zero Water Discharge Solutions in The Process Industry", Advance in Environmental Research, vol. 8, no. 2, 2003.

15 Bagajewicz M, Rodera H., and Savelski M. J., "Energy Efficient Water Utilization Systems in Process Plants", Computers \& Chemical Engineering, vol. 26, no. 1, 2002.

16 Rodera H., Savelski M. J., and Bagajewicz M., "Energy Retrofit with Simultaneous Optimization for a Crude Fractionation Unit", Latin American Applied Research, vol. 31, no. 5, 2001.

17 Farrell S., Hesketh R. P., Savelski M. J., and Slater C. S., "A model for collaboration between academia and industry", Proceedings of the $16^{\text {th }}$ International Congress of Chemical and Process Engineering, Session B4, Prague, Czech Republic, August 2004. 
18 Farrell, S. Hesketh, R.P., Savelski, M.J., Dahm, K., Slater, C. S., "Membrane projects with an industrial focus in the curriculum," Chemical Engineering Education, vol. 37, no. 1, 2003.

19 Pekula, N., B. Kuritz, J. Hearne, A. J. Marchese, and R. P. Hesketh, "The Effect of Ambient

Temperature, Humidity, and Engine Speed on Idling Emissions from Heavy-Duty Diesel Trucks," 2003

Transactions, Vol. 112, Section 4, Journal of Fuels and Lubricants, pages 148-158.

20 Newell, J. A., S. H. Farrell, R. P. Hesketh, and C. Stewart Slater, Introducing Emerging Technologies into the Curriculum through a Multidisciplinary Research Experience," Chemical Engineering Education 35(4) 296 Fall 2001.

21 Savelski M. J., Bagajewicz M. "On the Use of Linear Models for the Design of Water Utilization Systems in Refineries and Process Plants", Chemical Engineering Research and Design, 79, Part A, July 2001.

22 Jimenez-Gonzalez, C., Constable, D., Curzons. A.D., Cunningham, V. L., Developing GSK's Green Technology Guidance: Methodology for Case-Scenario Comparison of Technologies, Journal of Clean Technology and Environmental Policy, 4, 2002

23 Vit, J., Rowan Student Team Aims to Fight War on Pollution, The Plain Dealer, December 29, 2005.

24 Jolis, A. B., Students Lend a Hand with Cancer Drug, Gloucester County Times, A-1, A-5, December $28,2005$. 